\title{
Packet Loss Modeling for Perceptually Optimized 3D Transmission ${ }^{1}$
}

\author{
Irene Cheng, Lihang Ying and Anup Basu \\ Department of Computing Science, Univ. of Alberta, Edmonton, Alberta, Canada, Email: \{lin, lihang, anup\}@cs.ualberta.ca
}

\begin{abstract}
Transmissions over wireless and other unreliable networks can lead to packet loss. An area that has received limited research attention is how to tailor multimedia information taking into account the way packets are lost. We provide a brief overview of our research on designing a 3D perceptual quality metric integrating two important factors, resolution of texture and resolution of mesh, which control transmission bandwidth. We then suggest alternative strategies for packet 3D transmission of both texture and mesh. These strategies are then compared with respect to preserving 3D perceptual quality under packet loss in ad hoc wireless networks. Experiments are conducted to study how the time between consecutive packet transmission and packet size affects loss in wireless channels. A preliminary model for estimating the optimal packet size is then proposed.
\end{abstract}

\section{INTRODUCTION}

An important consideration in designing effective interactive online 3D systems is to adaptively adjust the model representation, while preserving satisfactory quality as perceived by a viewer. While most research in the literature focus on geometric compression and use only synthetic texture or color, we address both geometry resolution and realistic texture resolution, and analyze how these factors affect the overall perceptual quality. Our analysis is based on experiments conducted on human observers. The perceptual quality metric derived from experiments allows the appropriate level of detail (LOD) to be selected given the computation and bandwidth constraints. Detailed surveys on simplification algorithms can be found in $[1,2]$. These algorithms try to control the complexity of a mesh by developing various strategies for simplifying the LOD in different parts of a 3D object. In order to easily control the details on a 3D object we will follow a simple model approximation strategy based on multi-resolution representation of texture and mesh. An example of geometric simplification is shown in Figure 1, in which a Nutcracker toy model is simplified to various resolution levels (number of triangles is 1,260 left, 950 middle and 538 right).
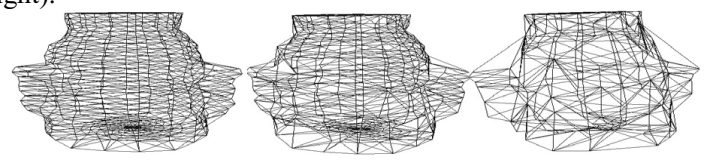

Figure 1: Nutcracker toy model at various mesh resolution levels.

One of the major drawbacks with most 3D transmission algorithms is that they do not consider the possibility of packet loss over wireless or unreliable networks. Some wireless protocols proposed in the last decade include Transmission Control Protocol (TCP), User Datagram Protocol (UDP), Indirect-TCP (I-TCP) [3], and so on. For wireless networks, where packet loss occurs as a result of unreliable links and route changes, the TCP strategy leads to further delays and degradation in transmission quality. Even though issues of multimedia transmission over wireless networks have received attention [4], relatively little work has been done addressing wireless 3D transmission. In recent research, approaches for robust transmission of mesh over wireless networks $[5,6]$ have been outlined. However, these methods do not take joint texture and mesh transmission into account. Also, in $[5,6]$ it is assumed that some parts of the mesh can be transmitted without loss over a wireless network allowing progressive mesh transmission to give good results. However, this assumption implies implementing a special standard with a combination of UDP and TCP protocols, which in general cannot be guaranteed in an arbitrary wireless environment. Special models for packet loss probability have been developed by other researchers [7]. However, these models are usually associated with requirements such as retransmission. To keep our study applicable in an unrestricted ad hoc wireless environment, we simply assume packet-based transmission where a certain percentage of the packets may be lost. In this scenario, we compare how various types of $3 \mathrm{D}$ transmission strategies fare, and how to take perceptual quality into account in designing a better strategy.

We consider an approach based on a perceptual quality metric following our earlier work in 2003 [8]. Other approaches to joint texture-mesh transmission have been discussed in $[9,10]$ in 2004. The approach in [9] is based on view-dependent rate-distortion optimization, whereas our approach is view-independent. Also, both $[9,10]$ are progressive, which necessitates greater protection of base layers in case of packet loss; our approach on the other hand does not need to guarantee delivery of certain packets in order to make other packets useful. Joint texture-mesh transmission of terrains was addressed in [11]; however, the author did not consider perceptual quality optimization.

The remainder of this paper is organized as follows: Section 2 reviews past work on perceptual quality evaluation and discusses how to relate bandwidth with texture and mesh reduction considering perceptual quality. Section 3 examines possible strategies for 3D image transmission and analyzes which one is most suitable for optimizing perceptual quality under packet loss. Some experimental results are outlined in Section 4. Experimental results on packet loss over a wireless LAN are summarized in Section 5. Following this, a strategy for packet size optimization is proposed in Section 6, before the work is concluded in Section 7.

\section{3D Perceptual QUality OPTIMIZation}

In the area of image compression, Mean Square Error (MSE) is commonly used as a quality predictor. However, past research has shown that MSE does not correlate well to perceived quality based on human evaluation [12]. Since this study, a number of new quality metrics based on the human visual system have been developed [13].

Several 3D objects were used as stimuli in our experiments. These objects were captured with the Zoomage 3D scanner. The participants (judges) were asked to compare the target stimulus with the two referential stimuli and assign it one of the following ratings: very poor (1), poor (2), fair (3), good (4), very good (5).

Figure 2 illustrates two referential stimuli (left and right) and one target stimulus (center) in the experiment.

Considering perceptual evaluations, we observed that:

(i) Perceived quality varies linearly with texture resolution (Fig. 3, left);

(ii) Perceived quality varies following an exponential curve for geometry (Fig. 3, right). (We consider an exponential, rather than a high degree polynomial, curve in order to have only a few parameters to estimate. Also, with several parameters in a polynomial there is likely to be significant variations in the parameters' values for small variations in the types of objects.)

Scaling the texture $(t)$ and geometry $(g)$ between 0 and 1 , it can be shown that:

$Q(g, t)=\frac{1}{\frac{1}{m+(M-m) t}+\left(\frac{1}{m}-\frac{1}{m+(M-m) t}\right)(1-g)^{c}}(1)$

\footnotetext{
${ }^{1}$ The support of Alberta Science and Research Authority (ASRA) and NSERC are gratefully acknowledged.
} 
Details of the perceptual evaluations and metric derivation can be found in our prior work [8]. Note that the quality value varies in the range of $1(\mathrm{~m})$ to $5(M)$, because of the range of values allowed in the perceptual ratings. Consider now that $b$ is the estimated total bandwidth for the transmission time interval, $T$ is the texture and $G$ is the geometry file sizes, possibly compressed, at maximum resolution. We assume that as the texture (or geometry) is scaled by a factor $t$ (or $g$ ) in both dimensions the corresponding file sizes get reduced to $t^{2} T$ (or $g^{2} G$ ). To utilize the bandwidth completely we must have: $b=t^{2} T+g^{2} \mathrm{G} \quad$ (2)

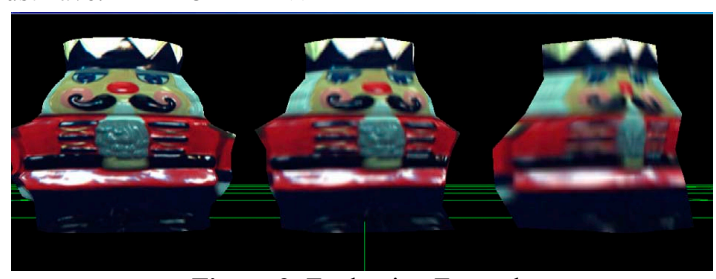

Figure 2: Evaluation Example

Given $b$ we can choose the relative proportion of texture and mesh to create a 3D model in many different ways, as long as Equation (2) is satisfied. The question is "What is the optimal choice maximizing perceptual quality?" Considering $\mathrm{m}=1, \mathrm{M}=5$, and $\mathrm{c}=2.7$ (approximately) for many objects based on perceptual tests, Equation (1) can be further simplified to:

$$
Q(g, t)=\frac{1}{\frac{1}{1+4 t}+\left(1-\frac{1}{1+4 t}\right)(1-g)^{2.7}}(3)
$$

Maximizing Equation (3) is equivalent to minimizing the inverse of this equation; considering this and Equation (2), optimizing quality reduces to minimizing:

$$
Q_{b, G, T}(t)=\frac{1}{1+4 t}+\left(1-\frac{1}{1+4 t}\right)\left(1-\sqrt{\frac{b-t^{2} T}{G}}\right)^{2.7}
$$

Where $b, G$ and $T$ are parameters.
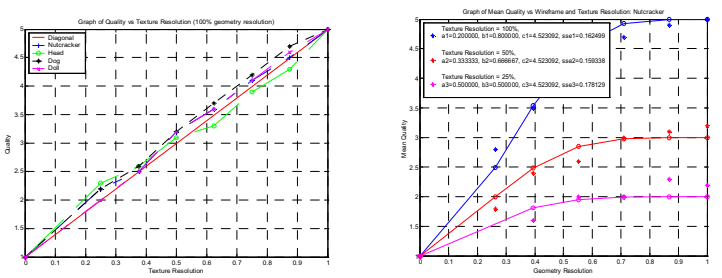

Figure 3: Left: Quality vs. Texture Resolution (100\% Geometry Resolution); Right: Quality vs. Geometry for various texture resolutions.

Example 1:

Let $\mathrm{b}=12$ Mbits, $\mathrm{T}=20 \mathrm{Mbits}$, and $\mathrm{G}=10 \mathrm{Mbits}$.

In this case $t$ can only vary in the range $[\sqrt{ } 2 / 20, \sqrt{ } 10 / 20]=[0.316, .707]$ so that Equation (2) can be satisfied. The graph of Equation (4) for varying $t$ for this case is shown in Figure 4. The optimal value of $t$ is close to 0.6 for this example. In general, given $T$ and $G$ for a $3 \mathrm{D}$ object, optimum $t$ can be pre-computed for a discrete number of $b$ values in the range $[0, T+G]$.

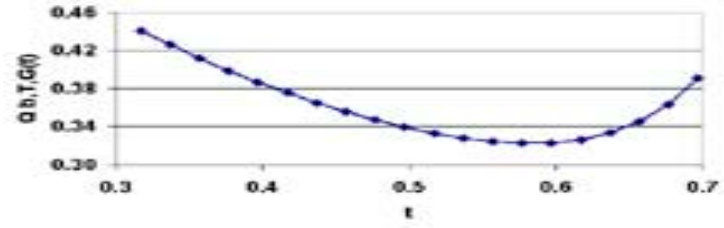

Figure 4: Inverse perceptual quality curve for Example 1.

\section{Perceptually OPTIMIZEd Transmission}

To simplify the model of wireless transmission, we assume that data is sent in packets of equal size and there is a possibility that a certain proportion of these packets may be lost. Various protocols [14] suggest re-transmission approaches in case of packet loss; however, re-transmission is not conducive to time bound real-time applications, such as $3 \mathrm{D}$ visualization for online games. We considered several possible strategies for packet construction in wireless 3D transmission, and then analyzed the pros and cons of each. We found that breaking up a 3D image into fragments can cause unacceptable voids; progressive transmission [15] necessitates receiving packets at lower levels before packets at higher levels can become useful; and sending duplicate copies of base layer packets in progressive transmission increases bandwidth requirements. We thus focus on the two following strategies, concentrating on regular mesh transmission.

\section{Strategy A:}

3d Partial Information Transmission (3PIT): In this approach we break up the texture and mesh into packets by sub-sampling into overlapping but non-identical components. At the client site the overall texture and mesh are reconstructed based on interpolation from the received packets. One implementation of this approach is given by the following algorithm:

\section{SERVER SITE:}

\section{T: original texture;}

M: original mesh, in a regular form allowing easy sub-sampling;

Construct $T_{1}, T_{2}, \ldots, T_{n}$ by regular, non-identical sub-sampling of $T$

(Comment: For example, given a $100 \times 100$ pixel texture $T$, we can

construct $T_{1}, T_{2}, \ldots, T_{16}$ by defining $T_{1}$ as $T(0+4 i, 0+4 j), i, j=0, \ldots 24 ; T_{2}$ as

$T(0+4 i, 1+4 j), i, j=0, \ldots 24 ; \ldots, T_{16}$ as $T(3+4 i, 3+4 j), i, j=0, \ldots 24$. $)$

Construct $M_{1}, M_{2}, \ldots, M_{n}$ by regular, non-identical sub-sampling of $M$;

Form packets $P_{1}, P_{2}, \ldots, P_{n}$ where $P_{i}=T_{i}+M_{i} ; i=1, \ldots, n$, with header and sub-sampling information added to each packet;

Transmit $n$ packets to a client on request, possibly in a randomized order; CLIENT SITE:

Request server to transmit a $3 D$ object;

Receive packets from server;

Uncompress mesh and texture data stored in this packet;

Set up initial display based on first packet received and interpolation information stored in header;

Update display based on next packet received:

Limitations of Strategy A:

One of the shortcomings of this approach is that the texture and mesh data receives equal importance; i.e., the same fraction of each is transmitted in a packet. The perceptual quality analysis in the last section shows that for optimizing perceptual quality the relative importance of texture and mesh can vary depending on the available bandwidth; this issue is not taken into account in Strategy A.

\section{Strategy B:}

3d Perceptually Optimized Partial Information Transmission (3POPIT): This approach extends 3PIT by taking perceptual quality into account. The algorithm modifies Strategy A by a bandwidth estimation step followed by perceptually optimized packet creation. Details are described below:

\section{SERVER SITE:}

T, $M$ : as for Strategy A;

Receive bandwidth estimate $\left(B_{e}\right)$ and estimated loss proportion (L) from requesting client;

Compute server transmitting bandwidth: $B_{s} \leftarrow B_{e} /(1-L)$;

Compute optimum texture and geometry scaling factors $t_{\mathrm{e}} \& g_{\mathrm{e}}$ following procedure for minimizing Equation (4) in the last section, considering bandwidth to be $B_{e}$;

Compute scaled texture $\left(T_{s}\right)$ and mesh $\left(G_{s}\right)$, assuming transmitting bandwidth $B_{s}$, based on factors $t_{\mathrm{e}} \& g_{\mathrm{e}}$;

(Comment: Specifically: $T s=\frac{t_{e}{ }^{2}}{(1-L)} T$ and $G s=\frac{g_{e}{ }^{2}}{(1-L)} G$; with texture and mesh possibly being interpolated to higher than the current maximum size in case the scaling factors are greater than 1.)

Construct $T_{s 1}, T_{s 2}, \ldots, T_{s n}$ by regular, non-identical sub-sampling of $T_{s}$; Construct $M_{s 1}, M_{s 2}, \ldots, M_{s n}$ by regular, non-identical sub-sampling of $M_{s}$; Form packets $P_{1}, P_{2}, \ldots, P_{n}$ where $P_{i}=T_{s i}+M_{s i} ; i=1, \ldots, n$, with header and sub-sampling information added to each packet; 
(Comment: Number of packets $n$ is chosen based on prior decision on packet size.)

Transmit $n$ packets to a client, possibly in a randomized order;

\section{CLIENT SITE}

Request server to transmit a $3 D$ object;

Receive packets from server for bandwidth estimation;

Estimate bandwidth $\left(B_{e}\right)$ based on number of packets received in a certain time interval and estimate loss proportion $(L)$;

Receive packets from server containing partial data on the $3 D$ object;

Uncompress mesh and texture data stored in this packet;

Set up initial display based on first packet received and interpolation

information stored in header;

Update display based on next packet received;

\section{Comments on Strategy B:}

On first observation it may appear that this strategy does not take packet loss proportion $(L)$ into account in the transmission strategy. However, in reality this is not the case. Without any packet loss, the transmission bandwidth $\left(B_{s}\right)$ would be used to compute the optimum texture and mesh scaling factors. When packets are lost the remaining packets may not be perceptually optimal for the effective bandwidth after packet loss. We thus form packets that are optimal at a lower bandwidth $\left(B_{e}\right)$.

One of the drawbacks of Strategy B is the need to estimate bandwidth and packet loss ratio. This estimation based transmission may not be practical where feedback from client to a server is not reliable, or for multicasting over heterogeneous networks with varying packet loss and bandwidths. This issue needs to be addressed in future research.

\section{ONLINE IMPLEMENTATION OF ALGORITHM}

We show some preliminary implementations towards deploying 3POPIT over a lossy wireless network. Figure 5 shows the effect of receiving and combining 2, 4 and 8 of 16 sub-samples of the nutcracker mesh. Note that results may vary from one execution to another for a random percentage of packet loss.

Figure 6 shows the effect of optimized vs. non-optimized transmission on perceptual quality. Two versions of the same model are shown, with the mesh on the left and the texture mapped on the right. Although the texture and mesh together for the left and right models use nearly the same bandwidth, 125 and $134 \mathrm{~Kb}$ respectively, the left one is favored by most viewers based on perceptual experiments.

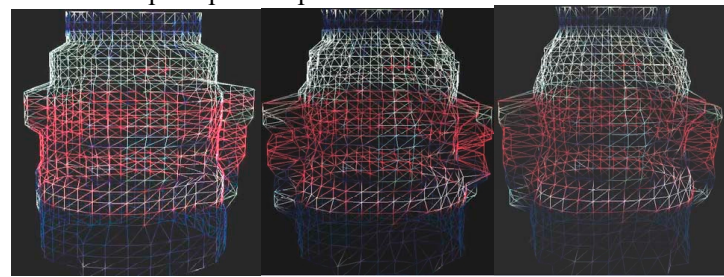

Figure 5: Interpolating and reconstructing mesh of nutcracker model when 2 (left), 4 (middle) and 8 of 16 packets are received.

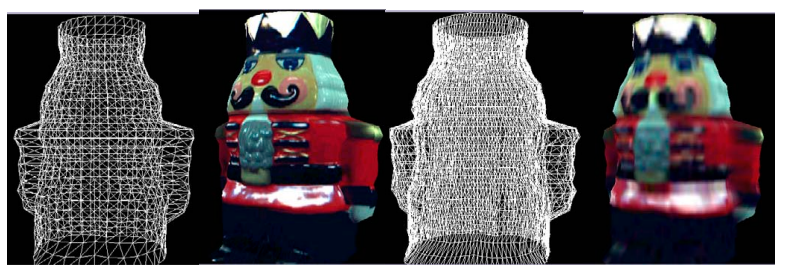

Figure 6: Two representations of the Nutcracker texture+mesh models: Left has lower quality mesh, requiring $125 \mathrm{~Kb}$ total bandwidth, but higher perceptual quality; Right has higher quality mesh, resulting in lower quality texture to keep total bandwidth at $134 \mathrm{~Kb}$, but has lower perceptual quality.

\section{NeTWORK EXPERIMENTS ON PACKET LOSS}

The long-term objective of our research in this area is to choose the appropriate parameters (packet size, sending interval, buffer size) for different applications to maximize throughput and minimize packet loss in different Internet environments with Wireless LAN access. In the experiments, the server side was a PC in the Department of Computing Science, University of Alberta, Edmonton, Canada. The client was a laptop, which linked to a router following $802.11 \mathrm{~b}$. The router accessed the Internet using Shaw Cable (with a maximum capacity of $600 \mathrm{KBps}$ ). The client was located in the same city as the server. Both client and server ran Red Hat Linux Release 9 Shrink (2.4.30 Kernel). We performed experiments in an environment with competing connections. In order to setup a competing environment, we configured the bandwidth of $802.11 \mathrm{~b}$ to $1 \mathrm{Mb}$. Another 4 FTP connections were added between the client and the server.

We first discuss the effect of socket buffer size on packet loss. With fixed packet size (4096 bytes) and sending rate (128KBps), the following table shows that different buffer sizes larger than the packet size make no significant difference on packet loss. However, if buffer size is less than packet size, all packets are lost. The interesting point is that when buffer size is equal to packet size, there is a significant packet loss as well.

\begin{tabular}{|l|l|l|}
\hline Buffer Size (bytes) & Receiving Throughtput (bps) & $\begin{array}{l}\text { Packet Loss } \\
(\%)\end{array}$ \\
\hline 32768 & 126611 & 0.98 \\
\hline 16384 & 127450 & 0.39 \\
\hline 4096 & 123138 & 3.91 \\
\hline 2048 & 0 & 100.00 \\
\hline
\end{tabular}

Next we look into how sending rate affects packet loss. We consider a large enough sending interval and fixed packet size (4096 Bytes), and let sending rate increase from 128 to $1024 \mathrm{KBps}$. It can be seen from Figure 7, that as sending rate increases, receiving rate increases and packet loss mostly remains nearly zero until around $500 \mathrm{Kbps}$. However, after sending rate overflows the connection (larger than $500 \mathrm{KBps}$ ), packet loss dramatically increases and receiving throughput drops owing to packet loss.

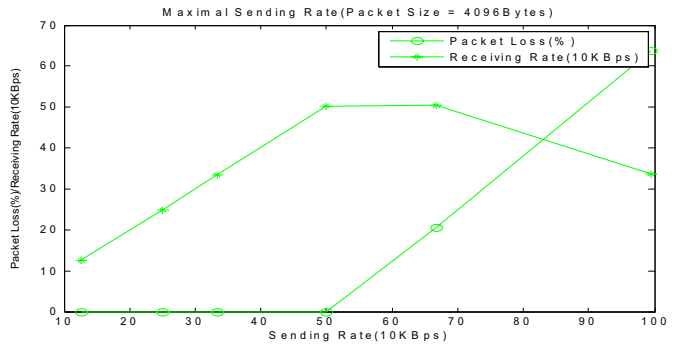

Figure 7: Effect of sending rate on packet loss.

Given a fixed sending rate $(64 \mathrm{KBps})$, packet sizes were selected from [128, $256,512,1024,2048,4096,8192,16384]$ bytes. Figure 8 shows some of our experimental results. On the left the packet loss is plotted against the time interval before transmitting the next packet. Clearly, the sending interval should not be too small $(<2 \mathrm{~ms})$ otherwise loss rate can be high. Fig. 8, right, shows how packet loss varies with packet size. When packet size is too small or too large, packet loss can be quite large. Packet loss was smallest when packet size was 1024 .
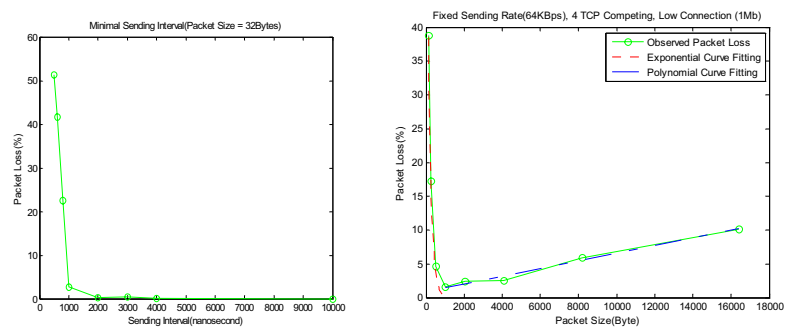

Figure 8: (Left) Packet loss vs. sending interval; (right) Packet loss vs. packet size in a congested network. 
In related work, [16] proposed to adjust the packet sending interval based on feedback from the network. But the work did not study the effect of different packet sizes. The authors proposed to determine the sending rate $T$ as a function of the packet size $s$, round-trip time $\boldsymbol{R}$, steady-state loss event rate $\boldsymbol{p}$, and the TCP retransmit timeout value $t_{\text {RTO }}$. But they did not study how the theoretical model works in a Wireless LAN (WLAN) environment. [17] studied UDP throughput and CPU utility for bulk data transfer with different sender and receiver buffer sizes and packet sizes. [18] studied the delay of UDP, TCP and TCP with the option NODELAY for voice applications sending 160 bytes per 20 milliseconds. Our work differs from others by carrying out a comprehensive study on packet size, sending interval, and buffer size through real world experiments in a competing WLAN environment, where there are packet losses besides congestion.

\section{Optimizing Packet Size}

In Figure 8, it can be observed that when packet size is small the loss can be quite high because of congestion resulting from the need to route many packets. As the packet size gradually increases beyond an optimum point with low loss, the loss rate increases again. We propose a strategy to model this characteristic and determine the optimal packet size following some simplifying assumptions. We assume exponential and linear models for packet loss depending on packet size; however, the approach can be extended to other models as well. Even though various models for packet loss over wireless networks have been proposed $[19,4]$, determining the optimal packet size has not received much attention. For simplicity, we use the following assumptions and notation:

$H$ : total header size for texture and mesh (e.g., JPEG + OBJ headers); $S$ : amount of payload data transmitted excluding header, if there is only 1 packet used;

$s_{n}$ : amount of payload data transmitted in each packet if $n$ packets used; Probability packet of size $\mathrm{D}$ is not lost $=e^{-\lambda D}$ : i.e., an exponential packet loss model is used with parameter $\lambda$ and packet size as variable.

Assuming a fixed transmitting bandwidth if amount of data transmitted is $S+H$ when only 1 packet is transmitted, then $s_{1}=S ; s_{2}=(S-H) / 2 ; \ldots ; s_{n}=$ $(S-(n-1) H) / n$

For large $n$, i.e. when $(n-1) / n \approx 1, s_{n} \approx S / n-H$.

Lemma 1: Following the model and assumptions above and independent transmission of packets, for large $n$ the total expected payload received with $n$ packets is: $f(n) \approx e^{\lambda H}\left[e^{-(\lambda S) / n}(S-n H)\right]$.

Proof: Follows from the definitions, and noting that the total expected payload received equals the sum of individual packet sizes times the probability that it is received. It is assumed that $(n-1) / n \approx 1$.

Theorem 1: The number of packets optimizing the expected amount of payload transmitted is an integer equal to either $\left\lfloor s \sqrt{\lambda^{2} H^{2}+4 \lambda H}-\frac{\lambda S}{2}\right\rfloor$ or

$\left\lceil s \sqrt{\lambda^{2} H^{2}+4 \lambda H}-\frac{\lambda S}{2}\right\rceil$

Proof: Follows from optimizing the function in Lemma 1 and the fact that the number of packets is an integer. Details of the proofs are not included because of limited space.

Now, suppose that the probability a packet of size $\mathrm{D}$ is not lost $=a-\lambda D$ : i.e., a linear packet loss model is used with parameter $\lambda$ and packet size as variable. This model is more meaningful in case the network characteristics follow the data in Figure 8. For this model it can be shown that:

Theorem 2: The number of packets optimizing the expected amount of payload transmitted for the linear model is an integer equal to either $\lfloor S \sqrt{\lambda /(a H)}\rfloor$ or $\lceil S \sqrt{\lambda /(a H)}\rceil$

Proof: Similar to proof Theorem 1, details skipped here.

For the data in Figure 8, we can observe that for the first part the curve fits a decreasing exponential function thus the optimum point is the rightmost point on this part. For the second part, after the minimum point, we can fit a linear function $y=0.8842+0.0006 x$. Thus the probability of a packet not lost equals $(1-y)=0.1058-0.0006 \mathrm{D}$; i.e., $a=0.1058$ and $\lambda=0.0006$ in Theorem 2. Given $S$ and $H$ we can determine the optimum number of packets following Theorem 2 for the linear section of the graph in Fig. 8.

\section{CONCLUSIONS AND FUtURE WORK}

In this paper, we discussed factors controlling 3D image degradation and outlined an approach for estimating perceptual quality considering variations in mesh and texture resolutions. A theoretical framework for determining the relative importance of texture $v s$. mesh was presented. An approach to optimizing perceptual quality under packet loss was then outlined. Experimental results were described to validate our approach. Finally, an approach for estimating the optimal packet size was proposed, following experimental results to collect real data on packet loss in congested wireless networks. In future work, we will extend and verify our packet size estimation method with more realistic models derived from tests over wireless networks to refine our assumptions. Implementations and user evaluations with handheld devices will also be conducted. Issues relating to connectivity transmission for arbitrary meshes, and MPEG4-3DMC compatibility [20], will be considered in our future work. We will also incorporate scale-space filtering [21] into our simplification approach.

\section{REFERENCES}

[1] P.S. Heckbert and M. Garland, "Survey of Polygonal Surface Simplification Algorithms," CMU, 1997.

[2] D. Luebke et al., "Level of Detail for 3D Graphics," Morgan Kaufmann, 2002.

[3] A. Bakre and B.R. Badrinath, "I-TCP: Indirect TCP for mobile hosts," Int. Conf. Distributed Computing Systems (ICDCS), 136-143, 1995.

[4] D. Wu and R. Negi, "Effective Capacity: A Wireless Channel Model for Support of QoS," IEEE Trans. on Wireless Communications, 2002.

[5] G. Alregib, Y. Altunbasak and J. Rossignac, "Error-resilient transmission of 3D Models," ACM Trans. on Graphics, April 05. (Early version in ICASSP 02.)

[6] Z. Chen, B. Bodenheimer and J.F. Barnes, "Robust transmission of 3D geometry over wireless networks," Web3D, pp: 161-172, 2003.

[7] K.V. Lee and S.T. Chanson, "Packet loss probability for real-time wireless communications," IEEE Trans. on Vehicular Technology, Nov. 2002.

[8] Y. Pan, I. Cheng and A. Basu, "Quality metric for approximating subjective evaluation of 3D objects," IEEE Trans. on Multimedia, April 2005. (Short version in IEEE International Conference on Image Processing 2003, Barcelona.)

[9] S. Yang et al. "Optimized mesh and texture multiplexing for progressive textured model transmission," ACM Multimedia, 2004

[10] D. Tian and G. AlRegib, "FQM: A fast quality measure for efficient transmission of textured 3D models," ACM Multimedia 2004.

[11] L. Balmelli, "Rate-distortion optimal mesh simplification and communication," Ph.D. dissertation, Ecole Polytechnique Federale de Lausanne, 2001.

[12] J. L. Mannos and D. J. Sakrison, "The Effects of a Visual Fidelity Criterion on Encoding of Images," IEEE Trans. on Information Theory, 1974.

[13] J. O. Limb, "Distortion Criteria of the Human Viewer," IEEE Transactions on SMC, Vol. 9, No.12, pp. 778-793, December 1979.

[14] R. Caceres and L. Iftode, "Improving the performance of reliable transport protocols in mobile computing environments," IEEE J. Select. Areas Comm., vol. 13, pp. 850-857, June 1995.

[15] H. Hoppe, "Progressive meshes," SIGGRPAH 1996, LA, USA.

[16] A. C. Feng, A. C. Kapadia, W. Frng, G. Belford, Packet Spacing - An Enabling Mechanism for Delivering Multimedia Content in Computational Grids, The Journal of Supercomputing, 23, 51-66, 2002

[17] Y. Gu and R. L. Grossman, Optimizing UDP-based Protocol Implementations, Workshop on Protocols for Fast Long-Distance Networks (PFLDNet), Feb. 2005.

[18] X. Zhang and H. Schulzrinne, Voice over TCP and UDP, Technical Report, Department of Computer Science,Columbia University, 2004

[19] R. El-Azouzi and E. Altman, "A queuing analysis of packet dropping over a wireless link with retransmissions," Proc. PWC, Italy, 2003.

[20] ISO/IEC 14496-2:2000, Amendment 1, "Coding of audio-visual objects - Part 2: Visual version 2," 2000.

[21] I. Cheng and P. Boulanger, "Feature extraction on 3D TexMesh using Scale-space analysis and Perceptual Evaluation," IEEE Trans. on CSVT, Special Issue on Scale-space Feature Extraction, Oct, 2005. 